\title{
Mitochondrial Heme Synthesis Enzymes as Therapeutic Targets in Vascular Diseases
}

\author{
Trupti Shetty ${ }^{1,2}$ and Timothy W. Corson ${ }^{1,2,3 *}$ \\ ${ }^{1}$ Department of Ophthalmology, Eugene and Marilyn Glick Eye Institute, Indiana University School of Medicine, Indianapolis, \\ IN, United States, ${ }^{2}$ Department of Pharmacology and Toxicology, Indiana University School of Medicine, Indianapolis, IN, \\ United States, ${ }^{3}$ Department of Biochemistry and Molecular Biology, Indiana University School of Medicine, Indianapolis, IN, \\ United States
}

Keywords: age-related macular degeneration, diabetic retinopathy, angiogenesis, neovascularization, ferrochelatase, electron transport chain, endothelial nitric oxide synthase, heme synthesis

\section{INTRODUCTION: SYNTHESIS AND FUNCTIONS OF HEME}

OPEN ACCESS

Edited by:

Zhongjie Fu,

Boston Children's Hospital and Harvard Medical School, United States

Reviewed by:

Yuqing Huo,

Augusta University, United States Keisuke Yanagida,

National Center For Global Health and Medicine, Japan

*Correspondence:

Timothy W. Corson tcorson@iu.edu

Specialty section:

This article was submitted to Neuropharmacology,

a section of the journal

Frontiers in Pharmacology

Received: 30 November 2019 Accepted: 23 June 2020 Published: 15 July 2020

Citation:

Shetty T and Corson TW (2020)

Mitochondrial Heme Synthesis Enzymes as Therapeutic Targets in

Vascular Diseases.

Front. Pharmacol. 11:1015. doi: 10.3389/fphar.2020.01015
Mitochondrial function in endothelial cells (EC) is interconnected by a mesh of signaling molecules that cross pathways often (Kluge et al., 2013). One such versatile biomolecule is heme. Heme is important for respiration, curbing oxidative stress, drug metabolism, and oxygen transport (Dailey and Meissner, 2013). The heme synthesis pathway and intermediates have been studied in detail over decades, with crystal structures and cloned genes available (Poulos, 2014). Intriguingly, heme is an important prosthetic moiety of key proteins of EC (Chiabrando et al., 2014b).

In mammalian cells, heme synthesis is accomplished in the mitochondria and cytosol over a series of eight enzymatic reactions, followed by modification of heme in a couple of sub-hemylation steps (Nilsson et al., 2009; Hamza and Dailey, 2012; Dailey et al., 2017). Heme biosynthesis in cells other than erythrocytes is initiated by the rate-limiting enzyme aminolevulinic acid synthase (ALAS1) that catalyzes formation of 5-aminolevulinic acid (ALA) from succinyl-CoA and glycine (Figure 1A). ALA is exported into the cytosol and converted via several intermediates into coproporphyrinogen-III (CPO) by coproporphyrinogen oxidase (CPOX); $\mathrm{CPO}$ is then transported back into the mitochondria for the last two steps of the pathway. In the final step, ferrochelatase $(\mathrm{FECH})$ incorporates ferrous iron into protoporphyrin IX (PPIX), synthesizing protoheme. Heme is then available to enable cellular processes by combining with enzyme subunits as a prosthetic group. For example, heme-iron is part of the catalytically active form of endothelial nitric oxide synthase (eNOS) (Raman et al., 1998). Similarly, different forms of heme are incorporated into mitochondrial respiratory complexes I-IV of the electron transport chain (ETC) (Kim et al., 2012). Of course, the majority of heme is used for incorporation into hemoglobin during erythropoiesis (Korolnek and Hamza, 2014) and some (primarily in the liver) for the synthesis of cytochrome P450s, responsible for xenobiotic metabolism (Correia et al., 2011).

Apart from being a prosthetic cofactor for enzymes, heme's regulated production ensures that active iron is sequestered before it can promote formation of reactive oxygen species (ROS) (Ryter and Tyrrell, 2000). Hence, heme plays a crucial role in ROS homeostasis in the mitochondria, without which many mitochondrial processes would be damaged (Alonso et al., 2003). One key regulator involved in detoxification of ROS and stimulating mitochondrial biogenesis is 


\section{^}
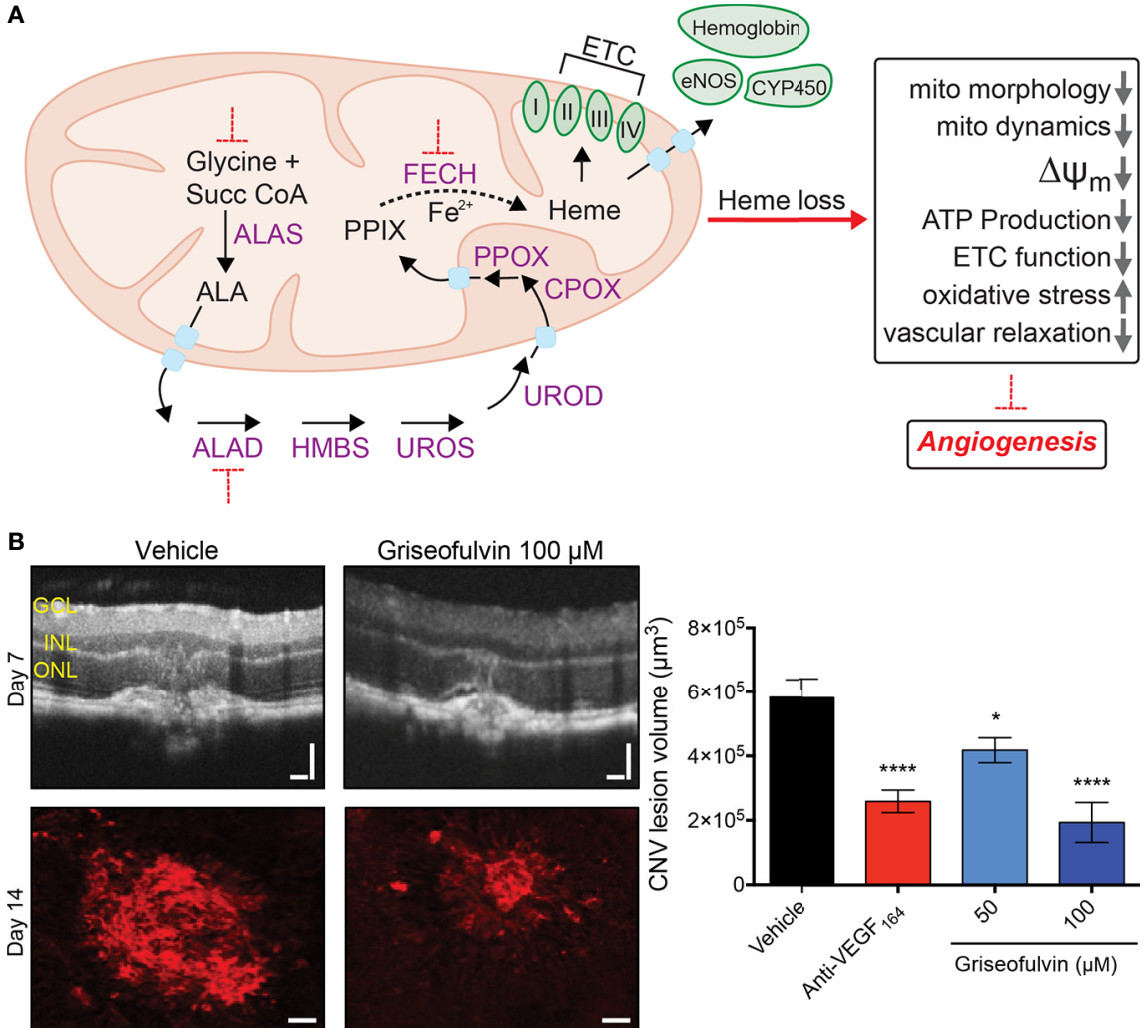

FIGURE 1 | Schematic diagram of the heme synthesis pathway in the mitochondrion and effect of Fech inhibition in vivo. (A) Eight sequential steps in the heme synthesis pathway are depicted, along with some heme-containing proteins. Red dotted lines indicate blockade. (B) Fech inhibition using griseofulvin in the laser-induced choroidal neovascularization (CNV) mouse model. CNV was confirmed by optical coherence tomography (OCT). Griseofulvin treated eyes had significantly smaller neovascular lesions as seen in red agglutinin staining for vasculature. Retinal layers indicated: GCL, ganglion cell layer; INL, inner nuclear layer, ONL, outer nuclear layer; Scale bars for OCT images and agglutinin immunostaining are 100 and $50 \mu \mathrm{m}$, respectively. ${ }^{*} \mathrm{p}=0.015 ;{ }^{* \star \star *} \mathrm{p}=0.0001$ versus vehicle, ANOVA with Dunnett's post hoc tests ( $\mathrm{n}=11-13$ eyes per group). Anti-VEGF 164 is a positive control antibody therapy. Figure modified from Basavarajappa et al., 2017 @ 2017 The Authors, CC BY 4.0. Succ CoA, succinyl-CoA, ALA, 5-aminolevulinic acid; ALAS, ALA synthase; ALAD, ALA dehydratase; HMBS, hydroxymethylbilane synthase; UROS, uroporphyrinogen synthase; UROD, uroporphyrinogen decarboxylase; CPOX, coproporphyrinogen oxidase; PPOX, protoporphyrinogen oxidase; FECH, ferrochelatase; PPIX, protoporphyrin IX; eNOS, endothelial nitric oxide synthase; CYP450, cytochrome P450; ETC, electron transport chain; $\Delta \Psi_{\mathrm{m}}=$ mitochondrial membrane potential; mito, mitochondria.

proliferator-activated receptor gamma coactivator $1 \alpha(\mathrm{PGC} 1 \alpha)$ (Austin and St-Pierre, 2012). PGC1 $\alpha$ regulates ALAS1 expression in the liver, linking heme synthesis directly to the nutritional state of cells (Handschin et al., 2005). Fastinginduced PGC1 $\alpha$ was found to be essential for vascular growth and pathological angiogenesis (Saint-Geniez et al., 2013). Here, we review recent studies that have identified an unexpected link between angiogenesis and heme synthesis, offering exciting therapeutic relevance to vascular diseases like retinopathy of prematurity (ROP), proliferative diabetic retinopathy (PDR), and wet age-related macular degeneration (AMD).

\section{HEME SYNTHESIS PROTEINS AS ANGIOGENESIS MEDIATORS}

The terminal heme synthesis enzyme, ferrochelatase, encoded by $\mathrm{FECH}$, was the first heme pathway component to be identified as a druggable target in pathological angiogenesis. FECH blockade (both genetically and pharmacologically) reduced proliferation, migration and endothelial tube formation in microvascular ECs. This effect was specific to ECs; FECH inhibition had a negligible effect on non-endothelial ocular cell proliferation. This antiangiogenic effect was also seen in vivo: mice with a partial loss-of-function $\mathrm{Fech}^{\mathrm{m} 1 \mathrm{Pas}}$ point mutation formed reduced neovascular lesions in the eye in the laser-induced choroidal neovascularization (L-CNV) model with features of wet AMD, as did mice with ocular Fech knockdown or inhibition (Figure 1B). In addition, FECH was overexpressed in and around these lesions, and in human wet age-related macular degeneration eyes (Basavarajappa et al., 2017). Moreover, FECH was upregulated, particularly in neovascular tufts, in the oxygeninduced retinopathy (OIR) mouse model of ROP (Pran Babu et al., 2020). The mechanisms of how heme contributes to EC physiology and drives angiogenesis are now beginning to be understood. 


\section{MECHANISMS OF HEME REGULATION OF ANGIOGENESIS}

\section{Mitochondrial Function}

Inhibition of heme synthesis has varying impact on the hemoproteins of the ETC (Vijayasarathy et al., 1999; Atamna et al., 2001). Heme $b$ and $c$ are present in complexes II and III, whereas complex IV has two groups of heme $a$, made after two consecutive modifications to protoheme (Kim et al., 2012). We recently showed that loss of heme via blockade of the terminal enzyme FECH in retinal ECs specifically causes complex IV dysfunction with negligible effects on other complexes of the ETC (Shetty et al., 2020). Complex IV protein and activity were significantly decreased by small molecule or genetic inhibition of FECH, but partially restored after heme supplementation. This loss in complex IV was accompanied by a depolarized mitochondrial membrane. Furthermore, heme depletion damaged both oxidative phosphorylation and glycolysis in retinal and choroidal ECs, along with a decrease in mitochondrial fusion and elevated ROS. This work characterized the direct effect of heme blockade on EC metabolism for the first time (Shetty et al., 2020).

Another recent study elucidated the contribution of the serine synthesis pathway to heme and EC metabolism (Vandekeere et al., 2018). Inhibition of the serine synthesis enzyme phosphoglycerate dehydrogenase (PHGDH) reduced glycine (substrate for the first step of the heme synthesis pathway), leading to an indirect decrease of heme enzymes and an eventual reduction in heme production in ECs. This also caused mitochondrial defects like reduced respiration, smaller mitochondria, increased fission, reduced fusion, and elevated mitophagy. Neonatal mice with silenced PHGDH had reduced retinal vascularization and reduced vessel area in the brain, heart, and kidney. Additionally, another group demonstrated that complex III is essential for EC proliferation (but not migration) in macrovascular ECs. Conditional knockout of EC-specific complex III led to reduced retinal, lung, and tumor neovascular blood vessels (Diebold et al., 2019). Loss of FECH activity was anti-proliferative for brain microvascular ECs, with no effect on macrovascular ECs (Basavarajappa et al., 2017). This was in contrast to reduced heme synthesis seen in macrovascular ECs as a result of aberrant serine synthesis (Vandekeere et al., 2018). The differential phenotypes of heme loss in microvasculature versus macrovasculature remain unclear and solicit further studies (Ghitescu and Robert, 2002; Sandoo et al., 2011).

Sprouting human umbilical vein ECs are highly glycolytic, producing up to $85 \%$ of ATP through the glycolysis pathway. During angiogenesis, endothelial tip and stalk cells dynamically switch their glycolytic activity depending on the energy demands of the tip cells and the proliferating stalk cells (De Bock et al., 2013). Recently, endothelial tip cells were reported to be less glycolytic during angiogenic cell differentiation (Yetkin-Arik et al., 2019), however more studies are warranted to validate such observations. Additionally, mitochondrial fatty acid oxidation has a role in proliferation of sprouting ECs (Schoors et al., 2015). While blocking heme production diminishes glycolytic capacity of retinal ECs (Shetty et al., 2020), it is as yet unclear whether heme regulation of EC metabolism varies between tip and non-tip ECs. Recent genomic analysis of murine choroidal ECs from neovascularization revealed potential metabolic candidates not found in healthy cells, suggesting targeting endothelial metabolism could be the way forward in vascular therapeutics (Rohlenova et al., 2020).

\section{Cytosolic Effects}

Lack of heme synthesis also leads to incomplete formation of eNOS and reduced activity (Feng, 2012). Heme depletion via FECH inhibition led to decreased expression, hemylation, and activity of eNOS in retinal microvascular ECs (Basavarajappa et al., 2017). Heme inhibition by chemically blocking the second synthesis enzyme aminolevulinic acid dehydratase (ALAD) in rats led to reduced eNOS and downstream mediator soluble guanylyl cyclase (sGC), both important in maintaining regular cardiovascular function. These effects did not affect vascular tension and resulted in no change to arterial blood pressure (Bourque et al., 2010). But heme depletion-driven eNOS dysfunction led to impaired NO mediated vascular relaxation in bovine coronary arteries (Zhang et al., 2018). NO, a potent vasodilator, is pro-angiogenic and NO itself is known to inhibit hemylation of extramitochondrial apo-hemoproteins (Waheed et al., 2010).

It is important to note that heme overload in ECs also leads to abnormal angiogenesis. Silencing of the heme transporter FLVCR1a led to intracellular heme accumulation in microvascular ECs, but not in macrovascular ECs. This heme accumulation in microvascular ECs led to impaired angiogenesis, damaged vessel formation and embryonic lethality in vivo (Petrillo et al., 2018). Heme toxicity has been investigated previously in hemolytic diseases like sickle cell disease and thalassemia, where heme scavengers are helpful in reducing heme-induced ROS accumulation (Vinchi et al., 2013). In nonsmall cell lung cancer, tumor cells had elevated heme synthesis activity, increasing respiratory function of the ETC and enhancing tumorigenic properties like migration and invasiveness (Sohoni et al., 2019). This suggests in addition to heme loss being anti-angiogenic, heme synthesis overdrive can increase mitochondrial function, but this remains to be validated in ECs. It would be interesting to investigate whether heme mediates EC metabolism in neovascularized tumors in a similar fashion and whether heme synthesis blockers could be valuable as cancer therapies.

\section{THERAPEUTIC POTENTIAL OF TARGETING HEME SYNTHESIS IN NEOVASCULARIZATION}

Current therapeutic strategies targeting mitochondria involve key functions like mitochondrial division (Cassidy-Stone et al., 2008), ROS formation (Dhanasekaran et al., 2004), and metabolism (Mather et al., 2001; Csiszar et al., 2009) for age-related neurodegenerative diseases like Alzheimer's, Parkinson's, and 
Huntington's (Lane et al., 2015). Meanwhile, anti-vascular endothelial growth factor (VEGF) therapies remain classic biologics used for neovascular diseases such as wet AMD, PDR, and multiple cancers (Jain, 2014). Until our and others' work described above, there was no rationale for targeting heme synthesis as neovascularization therapy. But given the specific antiproliferative effects of FECH blockade in microvascular ECs, FECH inhibitors like $N$-methylprotoporphyrin have demonstrated potential in targeting neovascular pathologies, both in vitro and in the OIR mouse model (Basavarajappa et al., 2017; Pran Babu et al., 2020). Novel, drug-like FECH inhibitors are also a possibility (Corson et al., 2019; Sishtla et al., 2019).

Repurposing existing drugs for pathological angiogenesis also holds promise towards this end. Griseofulvin, an FDA-approved anti-fungal drug, has a long-known off-target effect of FECH inhibition (Brady and Lock, 1992; Liu et al., 2015). It has antiangiogenic effects in retinal ECs, blocking proliferation, migration, and tube formation in vitro and reducing neovascularization in vivo comparable to intraocular antiVEGF treatment, in both OIR and L-CNV mouse models (Figure 1B) (Basavarajappa et al., 2017; Pran Babu et al., 2020). Isoniazid, an anti-mycobacterial drug, decreases FECH expression while upregulating ALAS1 (Brewer et al., 2019), and thus could be tested for potential anti-angiogenic activity in neovascularization models. Other inhibitors of heme synthesis used in vitro include succinylacetone and salicylic acid that block ALAD and FECH respectively (Giger and Meyer, 1983; Gupta et al., 2013), however their use in preclinical angiogenesis models remains to be investigated.

Targeting mitochondrial proteins directly involved in ETC activity has limitations as well, with a direct consequence on mitochondrial function. However, extracellular supplementation of hemin (a more stable form of heme) is able to normalize some of the mitochondrial physiology, like eNOS levels, complex IV activity, and ETC function (Basavarajappa et al., 2017; Vandekeere et al., 2018; Shetty et al., 2020). Effect of FECH blockade can be titrated, with a dose dependent decrease in angiogenesis features observed in animal models and ECs in culture. Homozygous Fech $^{\mathrm{m} 1 \mathrm{Pas}}$ mice have significantly reduced neovascular lesions, compared to heterozygous $\mathrm{Fech}^{\mathrm{m} 1 \mathrm{Pas}}$ mice. And heterozygotes themselves have reduced lesions compared to wild-type (Basavarajappa et al., 2017), suggesting a window of FECH antiangiogenic effects without toxicity. However, complete loss of Fech and Alas1 are embryonically lethal to mice (Magness et al., 2002; Chiabrando et al., 2014a), highlighting the importance of modulating heme inhibition carefully.

Oral supplementation of heme, while still achieving therapeutic antiangiogenic effects of inhibitors, could be considered (Luan et al., 2017). In order to limit systemic toxicity, it would be helpful to localize therapeutic formulations to pathological tissue wherever possible. For example, in ocular neovascularization, therapeutic agents could be delivered through intravitreal or subretinal injection (Basavarajappa et al., 2017), or even as eyedrops if formulation allows; this is a promising area for future work. Therapeutic targeting specific to ECs could be included in drug delivery systems (Kawahara et al., 2013), since systemic deficiency in heme synthesis enzymes can lead to porphyrias. For example, erythropoietic protoporphyria is caused by toxic buildup of PPIX (Gouya et al., 1999). The phototoxic PPIX can be detrimental to cells, and is manipulated in photodynamic therapy (PDT) (Krammer and Plaetzer, 2008). However, it is unlikely that PPIX itself mediates anti-angiogenic effects, as ALA-PDT relies heavily on uptake of ALA (Wachowska et al., 2011). Moreover, as noted, hemin is able to rescue anti-angiogenic effects in ECs, even in the presence of PPIX build-up, suggesting that this mechanism is heme dependent and not due to PPIX toxicity.

\section{CONCLUSIONS AND FUTURE PROSPECTS}

Targeting intracellular heme, either via inhibition of synthesis through intermediary enzymes or blocking heme transport (through FLVCR) provides for a novel therapeutic strategy, one that is primed to be explored in detail in vascular biology. Key questions that need to be addressed are: Is the role of heme in angiogenesis limited to ETC and eNOS or do other hemecontaining proteins aid in anti-angiogenic effects? Which enzymes in the heme synthesis pathway are the most effectively targetable for treating pathological angiogenesis? What are the key differences in microvascular and macrovascular heme synthesis, and can we manipulate these therapeutically? Proliferative ECs appear to be particularly sensitive to heme loss, but is this sensitivity only relevant in vascular tissues? Most importantly, we also need to elucidate the contribution of heme and heme pathway intermediates in maintaining normal endothelial cellular physiology, to devise better strategies for future therapeutic interventions.

\section{AUTHOR CONTRIBUTIONS}

TS, TC: wrote the paper, edited the paper, and approved final version.

\section{FUNDING}

Related work in the Corson laboratory is supported by NIH/NEI R01EY025641, NIH/NCATS UL1TR001108, the Retina Research Foundation, the International Retinal Research Foundation, the BrightFocus Foundation, the Carl Marshall and Mildred Almen Reeves Foundation, and the Ralph and Grace Showalter Research Trust.

\section{ACKNOWLEDGMENTS}

We thank members of the Corson laboratory for comments on the manuscript. 


\section{REFERENCES}

Alonso, J. R., Cardellach, F., Lopez, S., Casademont, J., and Miro, O. (2003). Carbon monoxide specifically inhibits cytochrome $\mathrm{c}$ oxidase of human mitochondrial respiratory chain. Pharmacol. Toxicol. 93, 142-146. doi: 10.1034/j.1600-0773.2003.930306.x

Atamna, H., Liu, J., and Ames, B. N. (2001). Heme deficiency selectively interrupts assembly of mitochondrial complex IV in human fibroblasts: Relevance to aging. J. Biol. Chem. 276, 48410-48416. doi: 10.1074/jbc.M108362200

Austin, S., and St-Pierre, J. (2012). PGC1 $\alpha$ and mitochondrial metabolism: Emerging concepts and relevance in ageing and neurodegenerative disorders. J. Cell Sci. 125, 4963-4971. doi: 10.1242/jcs.113662

Basavarajappa, H. D., Sulaiman, R. S., Qi, X., Shetty, T., Sheik Pran Babu, S., Sishtla, K. L., et al. (2017). Ferrochelatase is a therapeutic target for ocular neovascularization. EMBO Mol. Med. 9, 786-801. doi: 10.15252/ emmm.201606561

Bourque, S. L., Benjamin, C. D., Adams, M. A., and Nakatsu, K. (2010). Lack of hemodynamic effects after extended heme synthesis inhibition by succinylacetone in rats. J. Pharmacol. Exp. Ther. 333, 290-296. doi: 10.1124/ jpet.109.162966

Brady, A. M., and Lock, E. A. (1992). Inhibition of ferrochelatase and accumulation of porphyrins in mouse hepatocyte cultures exposed to porphyrinogenic chemicals. Arch. Toxicol. 66, 175-181. doi: 10.1007/ BF01974011

Brewer, C. T., Yang, L., Edwards, A., Lu, Y., Low, J., Wu, J., et al. (2019). The isoniazid metabolites hydrazine and pyridoxal isonicotinoyl hydrazone modulate heme biosynthesis. Toxicol. Sci. 168, 209-224. doi: 10.1093/toxsci/ kfy294

Cassidy-Stone, A., Chipuk, J. E., Ingerman, E., Song, C., Yoo, C., Kuwana, T., et al. (2008). Chemical inhibition of the mitochondrial division dynamin reveals its role in Bax/Bak-dependent mitochondrial outer membrane permeabilization. Dev. Cell 14, 193-204. doi: 10.1016/j.devcel.2007.11.019

Chiabrando, D., Mercurio, S., and Tolosano, E. (2014a). Heme and erythropoiesis: More than a structural role. Haematologica 99, 973-983. doi: 10.3324/ haematol.2013.091991

Chiabrando, D., Vinchi, F., Fiorito, V., Mercurio, S., and Tolosano, E. (2014b). Heme in pathophysiology: A matter of scavenging, metabolism and trafficking across cell membranes. Front. Pharmacol. 5, 61. doi: 10.3389/fphar.2014.00061

Correia, M. A., Sinclair, P. R., and De Matteis, F. (2011). Cytochrome P450 regulation: The interplay between its heme and apoprotein moieties in synthesis, assembly, repair, and disposal. Drug Metab. Rev. 43, 1-26. doi: $10.3109 / 03602532.2010 .515222$

Corson, T. W., Seo, S. Y., Lee, B., and Sishtla, K. (2019). Ferrochelatase inhibitors and methods of use, International Patent Application PCT/US19/29909.

Csiszar, A., Labinskyy, N., Pinto, J. T., Ballabh, P., Zhang, H., Losonczy, G., et al. (2009). Resveratrol induces mitochondrial biogenesis in endothelial cells. Am. J. Physiol. Heart Circ. Physiol. 297, H13-H20. doi: 10.1152/ajpheart.00368.2009

Dailey, H. A., and Meissner, P. N. (2013). Erythroid heme biosynthesis and its disorders. Cold Spring Harb. Perspect. Med. 3, a011676. doi: 10.1101/ cshperspect.a011676

Dailey, H. A., Dailey, T. A., Gerdes, S., Jahn, D., Jahn, M., O’Brian, M. R., et al. (2017). Prokaryotic heme biosynthesis: Multiple pathways to a common essential product. Microbiol. Mol. Biol. Rev. 81, e00048-16. doi: 10.1128/ MMBR.00048-16

De Bock, K., Georgiadou, M., Schoors, S., Kuchnio, A., Wong, B. W., Cantelmo, A. R., et al. (2013). Role of PFKFB3-driven glycolysis in vessel sprouting. Cell 154, 651-663. doi: 10.1016/i.cell.2013.06.037

Dhanasekaran, A., Kotamraju, S., Kalivendi, S. V., Matsunaga, T., Shang, T., Keszler, A., et al. (2004). Supplementation of endothelial cells with mitochondria-targeted antioxidants inhibit peroxide-induced mitochondrial iron uptake, oxidative damage, and apoptosis. J. Biol. Chem. 279, 37575-37587. doi: $10.1074 /$ jbc.M404003200

Diebold, L. P., Gil, H. J., Gao, P., Martinez, C. A., Weinberg, S. E., and Chandel, N. S. (2019). Mitochondrial complex III is necessary for endothelial cell proliferation during angiogenesis. Nat. Metab. 1, 158-171. doi: 10.1038/s42255-018-0011-x

Feng, C. (2012). Mechanism of nitric oxide synthase regulation: electron transfer and interdomain interactions. Coord. Chem. Rev. 256, 393-411. doi: 10.1016/ j.ccr.2011.10.011
Ghitescu, L., and Robert, M. (2002). Diversity in unity: The biochemical composition of the endothelial cell surface varies between the vascular beds. Microsc. Res. Tech. 57, 381-389. doi: 10.1002/jemt.10091

Giger, U., and Meyer, U. A. (1983). Effect of succinylacetone on heme and cytochrome P450 synthesis in hepatocyte culture. FEBS Lett. 153, 335-338. doi: 10.1016/0014-5793(83)80637-1

Gouya, L., Puy, H., Lamoril, J., Da Silva, V., Grandchamp, B., Nordmann, Y., et al. (1999). Inheritance in erythropoietic protoporphyria: A common wild-type ferrochelatase allelic variant with low expression accounts for clinical manifestation. Blood 93, 2105-2110. doi: 10.1182/blood.V93.6.2105. 406k28_2105_2110

Gupta, V., Liu, S., Ando, H., Ishii, R., Tateno, S., Kaneko, Y., et al. (2013). Salicylic acid induces mitochondrial injury by inhibiting ferrochelatase heme biosynthesis activity. Mol. Pharmacol. 84, 824-833. doi: 10.1124/ mol.113.087940

Hamza, I., and Dailey, H. A. (2012). One ring to rule them all: trafficking of heme and heme synthesis intermediates in the metazoans. Biochim. Biophys. Acta 1823, 1617-1632. doi: 10.1016/j.bbamcr.2012.04.009

Handschin, C., Lin, J., Rhee, J., Peyer, A. K., Chin, S., Wu, P. H., et al. (2005). Nutritional regulation of hepatic heme biosynthesis and porphyria through PGC-1 $\alpha$. Cell 122, 505-515. doi: 10.1016/j.cell.2005.06.040

Jain, R. K. (2014). Antiangiogenesis strategies revisited: from starving tumors to alleviating hypoxia. Cancer Cell 26, 605-622. doi: 10.1016/j.ccell.2014.10.006

Kawahara, H., Naito, H., Takara, K., Wakabayashi, T., Kidoya, H., and Takakura, N. (2013). Tumor endothelial cell-specific drug delivery system using apelinconjugated liposomes. PloS One 8, e65499. doi: 10.1371/journal.pone.0065499

Kim, H. J., Khalimonchuk, O., Smith, P. M., and Winge, D. R. (2012). Structure, function, and assembly of heme centers in mitochondrial respiratory complexes. Biochim. Biophys. Acta 1823, 1604-1616. doi: 10.1016/ j.bbamcr.2012.04.008

Kluge, M. A., Fetterman, J. L., and Vita, J. A. (2013). Mitochondria and endothelial function. Circ. Res. 112, 1171-1188. doi: 10.1161/CIRCRESAHA.111.300233

Korolnek, T., and Hamza, I. (2014). Like iron in the blood of the people: The requirement for heme trafficking in iron metabolism. Front. Pharmacol. 5, 126. doi: 10.3389/fphar.2014.00126

Krammer, B., and Plaetzer, K. (2008). ALA and its clinical impact, from bench to bedside. Photochem. Photobiol. Sci. 7, 283-289. doi: 10.1039/B712847A

Lane, R. K., Hilsabeck, T., and Rea, S. L. (2015). The role of mitochondrial dysfunction in age-related diseases. Biochim. Biophys. Acta 1847, 1387-1400. doi: 10.1016/j.bbabio.2015.05.021

Liu, K., Yan, J., Sachar, M., Zhang, X., Guan, M., Xie, W., et al. (2015). A metabolomic perspective of griseofulvin-induced liver injury in mice. Biochem. Pharmacol. 98, 493-501. doi: 10.1016/j.bcp.2015.09.002

Luan, Y., Zhang, F., Cheng, Y., Liu, J., Huang, R., Yan, M., et al. (2017). Hemin improves insulin sensitivity and lipid metabolism in cultured hepatocytes and mice fed a high-fat diet. Nutrients 9, 805. doi: 10.3390/nu9080805

Magness, S. T., Maeda, N., and Brenner, D. A. (2002). An exon 10 deletion in the mouse ferrochelatase gene has a dominant-negative effect and causes mild protoporphyria. Blood 100, 1470-1477. doi: 10.1182/blood-2001-12-0283

Mather, K. J., Verma, S., and Anderson, T. J. (2001). Improved endothelial function with metformin in type 2 diabetes mellitus. J. Am. Coll. Cardiol. 37, 1344-1350. doi: 10.1016/S0735-1097(01)01129-9

Nilsson, R., Schultz, I. J., Pierce, E. L., Soltis, K. A., Naranuntarat, A., Ward, D. M., et al. (2009). Discovery of genes essential for heme biosynthesis through largescale gene expression analysis. Cell Metab. 10, 119-130. doi: 10.1016/ j.cmet.2009.06.012

Petrillo, S., Chiabrando, D., Genova, T., Fiorito, V., Ingoglia, G., Vinchi, F., et al. (2018). Heme accumulation in endothelial cells impairs angiogenesis by triggering paraptosis. Cell Death Differ. 25, 573-588. doi: 10.1038/s41418017-0001-7

Poulos, T. L. (2014). Heme enzyme structure and function. Chem. Rev. 114, 39193962. doi: $10.1021 / \mathrm{cr} 400415 \mathrm{k}$

Pran Babu, S. P. S., White, D., and Corson, T. W. (2020). Ferrochelatase regulates retinal neovascularization. FASEB J. in press. doi: 10.1096/fj.202000964R

Raman, C. S., Li, H., Martasek, P., Kral, V., Masters, B. S., and Poulos, T. L. (1998). Crystal structure of constitutive endothelial nitric oxide synthase: A paradigm for pterin function involving a novel metal center. Cell 95, 939-950. doi: $10.1016 /$ S0092-8674(00)81718-3 
Rohlenova, K., Goveia, J., Garcia-Caballero, M., Subramanian, A., Kalucka, J., Treps, L., et al. (2020). Single-cell RNA sequencing maps endothelial metabolic plasticity in pathological angiogenesis. Cell Metab. 31, 862-877 e814. doi: 10.1016/j.cmet.2020.03.009

Ryter, S. W., and Tyrrell, R. M. (2000). The heme synthesis and degradation pathways: role in oxidant sensitivity. Heme oxygenase has both pro- and antioxidant properties. Free Radic. Biol. Med. 28, 289-309. doi: 10.1016/S08915849(99)00223-3

Saint-Geniez, M., Jiang, A., Abend, S., Liu, L., Sweigard, H., Connor, K. M., et al. (2013). PGC-1 $\alpha$ regulates normal and pathological angiogenesis in the retina. Am. J. Pathol. 182, 255-265. doi: 10.1016/j.ajpath.2012. 09.003

Sandoo, A., Carroll, D., Metsios, G. S., Kitas, G. D., and Veldhuijzen Van Zanten, J. J. (2011). The association between microvascular and macrovascular endothelial function in patients with rheumatoid arthritis: a cross-sectional study. Arthritis Res. Ther. 13, R99. doi: 10.1186/ar3374

Schoors, S., Bruning, U., Missiaen, R., Queiroz, K. C., Borgers, G., Elia, I., et al. (2015). Fatty acid carbon is essential for dNTP synthesis in endothelial cells. Nature 520, 192-197. doi: 10.1038/nature14362

Shetty, T., Sishtla, K., Park, B., Repass, M. J., and Corson, T. W. (2020). Heme synthesis inhibition blocks angiogenesis via mitochondrial dysfunction. iScience. in press. Preprint: bioRxiv. 836304. doi: 10.1101/836304.

Sishtla, K., Lee, S., Lee, J. ,. E., Seo, S. Y., and Corson, T. W. (2019). Discovery of ferrochelatase inhibitors as antiangiogenic agents. Invest. Ophthalmol. Vis. Sci. 60, E-abstract 5405.

Sohoni, S., Ghosh, P., Wang, T., Kalainayakan, S. P., Vidal, C., Dey, S., et al. (2019). Elevated heme synthesis and uptake underpin intensified oxidative metabolism and tumorigenic functions in non-small cell lung cancer cells. Cancer Res. 79, 2511-2525. doi: 10.1158/0008-5472.CAN-18-2156

Vandekeere, S., Dubois, C., Kalucka, J., Sullivan, M. R., Garcia-Caballero, M., Goveia, J., et al. (2018). Serine synthesis via PHGDH is essential for heme production in endothelial cells. Cell Metab. 28, 573-587 e513. doi: 10.1016/ j.cmet.2018.06.009

Vijayasarathy, C., Damle, S., Lenka, N., and Avadhani, N. G. (1999). Tissue variant effects of heme inhibitors on the mouse cytochrome $c$ oxidase gene expression and catalytic activity of the enzyme complex. Eur. J. Biochem. 266, 191-200. doi: 10.1046/j.1432-1327.1999.00843.x

Vinchi, F., De Franceschi, L., Ghigo, A., Townes, T., Cimino, J., Silengo, L., et al. (2013). Hemopexin therapy improves cardiovascular function by preventing heme-induced endothelial toxicity in mouse models of hemolytic diseases. Circulation 127, 1317-1329. doi: 10.1161/CIRCULATIONAHA.112.130179

Wachowska, M., Muchowicz, A., Firczuk, M., Gabrysiak, M., Winiarska, M., Wańczyk, M., et al. (2011). Aminolevulinica acid (ALA) as a prodrug in photodynamic therapy of cancer. Molecules 16 (5), 4140-4164. doi: 10.3390/ molecules 16054140

Waheed, S. M., Ghosh, A., Chakravarti, R., Biswas, A., Haque, M. M., Panda, K., et al. (2010). Nitric oxide blocks cellular heme insertion into a broad range of heme proteins. Free Radic. Biol. Med. 48, 1548-1558. doi: 10.1016/ j.freeradbiomed.2010.02.038

Yetkin-Arik, B., Vogels, I. M. C., Neyazi, N., Van Duinen, V., Houtkooper, R. H., Van Noorden, C. J. F., et al. (2019). Endothelial tip cells in vitro are less glycolytic and have a more flexible response to metabolic stress than non-tip cells. Sci. Rep. 9, 10414. doi: 10.1038/s41598-019-46503-2

Zhang, B., Alruwaili, N., Kandhi, S., Deng, W., Huang, A., Wolin, M. S., et al. (2018). Inhibition of ferrochelatase impairs vascular eNOS/NO and sGC/ cGMP signaling. PloS One 13, e0200307. doi: 10.1371/journal.pone.0200307

Conflict of Interest: TC is a named inventor on patent applications related to this topic.

The remaining author declares that the research was conducted in the absence of any commercial or financial relationships that could be construed as a potential conflict of interest.

Copyright (c) 2020 Shetty and Corson. This is an open-access article distributed under the terms of the Creative Commons Attribution License (CC BY). The use, distribution or reproduction in other forums is permitted, provided the original author(s) and the copyright owner(s) are credited and that the original publication in this journal is cited, in accordance with accepted academic practice. No use, distribution or reproduction is permitted which does not comply with these terms. 